\title{
Esquistossomose mansoni em duas mesorregiões do Estado de Alagoas
}

\author{
Schistosomiasis mansoni in two mesoregions \\ of the State of Alagoas
}

\author{
Janira Lúcia Assumpção Couto ${ }^{1}$
}

\begin{abstract}
RESUM0
№ Brasil, índices elevados da esquistossomose mansônica correspondem, na grande maioria dos casos, à presença da espécie Biomphalaria glabrata, principal transmissora do Schistosoma mansoni, nas localidades endêmicas. Foi realizado estudo em 40 municípios endêmicos do Estado de Alagoas, com 0 objetivo de verificar a existência da espécie e sua importância na manutenção da esquistossomose nesse Estado. Desses municípios, 28 são pertencentes à mesorregião do leste Alagoano e 12 à mesorregião do Agreste Alagoano. Os moluscos procederam de diversos tipos de criadouros: riachos, córregos, valas, açudes, brejos e poços. As coletas foram realizadas no período de fevereiro de 1996 a dezembro de 1998. Para a identificação de Biomphalaria glabrata, foi efetuado o exame anatômico das partes moles, após remoção das conchas. Adetecção de cercárias do Schistosoma mansoni foi realizada através da técnica de esmagamento, calculandose o percentual de infecção. Em 32 (80\%) dos municípios estudados foi encontrada Biomphalaria glabrata, seis deles apresentando moluscos infectados com cercárias do parasita. Penedo apresentou a maior (6,6\%) taxa de infecção, seguindo-se Ibateguara (5,6\%). Taxas menores foram observadas em Chã Preta (2,7\%), em Murici (2,5\%), Porto Real do Colégio (0,1\%) e Igreja Nova (0,1\%). 0 inquérito copro-parasitológico efetuado pela Fundação Nacional de Saúde em 1997, 1998/1999 e 2000, confirmou a importância da endemia nessas regiões do Estado.
\end{abstract}

Palavras-chaves: Esquistossomose. Biomphalaria glabrata. Epidemiologia. Alagoas.

\begin{abstract}
In Brazil, high levels of schistosomiasis mansoni are linked to the presence of the species Biomphalaria glabrata, considered to be the main host of Schistosoma mansoni in endemic areas. This work conducted a survey of 40 endemic municipalities in the State of Alagoas, aiming at identifying this species and its important role in the maintenance of schistosomiasis. Among the municipalities surveyed, 28 lie in the mesoregion of the Leste Alagoano and 12, in the mesoregion of the Agreste Alagoano. The snails collected for analysis came from different types of habitats, namely streams, springs, ditches, dams, swamps, wells and ponds. The snails were collected from February 1996 through December 1998. The identification of Biomphalaria glabrata was made through anatomical analysis of the soft inner parts of the snails after having been removed their shells. As for the detection of cercariae of Schistosoma mansoni, the crush technique was applied in order to calculate the percentage of infection rates. Among the areas surveyed, 32 municipalities ( $80 \%$ of the total), presented the species Biomphalaria glabrata, six of which containing snails infected with cercariae of the parasite. Penedo presented the highest infection rate (6.6\%), followed by Ibateguara (5.6\%). Lower rates were noticed in Chã Preta (2.7\%), in Murici (2.5\%), Porto Real do Colégio (0.1\%) and Igreja Nova (0.1\%).The coproparasitologic analyses conducted at the Fundação Nacional de Saúde in 1997, 1998/1999 and 2000, confirmed the importance of schistosomiasis mansoni in the areas surveyed in this work.
\end{abstract}

Key-words: Schistosomiasis. Biomphalaria glabrata. Epidemiology. Alagoas.

1. Departamento de Patologia do Centro de Ciências Biológicas da Universidade Federal de Alagoas, Maceió, AL Apoio da Fundação Nacional de Saúde (Programa de Controle da Esquistossomose).

Endereço para correspondência: Profạ. Janira Lúcia A. Couto. R. Desp. Humberto Guimarães 191/102, Ponta Verde, 57035-030 Maceió, AL, Brasil. e-mail: janelu@ neoline.com.br

Recebido para publicação em 26/2/2004

Aceito em 18/3/2005 
Aesquistossomose mansônica (EM) continua a ser um grave problema de Saúde Pública no Brasil, com áreas de concentração no Nordeste ${ }^{233233}$ e no Estado de Minas Gerais ${ }^{4567}$. A endemia tem possibilidades de expandir-se em decorrência da associação de fatores, tais como as vastas regiões agrícolas com extensivos projetos de irrigação ${ }^{940}$, a conseqüente devastação ambiental e a ocupação da terra por migrantes de baixo nível sócioeconômico, fatores estes que estimulam a proliferação dos moluscos transmissores da doença ${ }^{30}$.

A presença de Biomphalaria glabrata (Say, 1818), em determinadas localidades, associada à presença de indivíduos com esquistossomose, pode estabelecer a endemia esquistossomótica. A espécie é o mais importante vetor do Schistosoma mansoni ( Sambom, 1907) nas Américas, devido ao alto potencial biológico de infecção natural e vasta distribuiçãa $0^{1252638}$.

№ Brasil, a dominância de B. glabrata abrange a Região Nordeste, ao longo da faixa costeira e áreas interiores adjacentes dos Estados do Rio Grande do Norte, Paraíba, Pernambuco, Alagoas, Sergipe, até 0 sudeste da Bahia. Na Região Sudeste, atinge a parte de Minas Gerais, a leste do rio São Francisco, e 0 norte do Espírito Santo; existem, ainda, focos periféricos isolados deste molusco no Maranhão, Pará, Goiás, São Paulo e Paraná26, e recentemente no Rio Grande do Sul ${ }^{8}$.

Em Alagoas, os primeiros registros de sua ocorrência foram efetuados por Lucena na Cidade de Penedo, onde encontrou planorbídeos infectados ${ }^{19}$. Posteriormente, a espécie foi descoberta em mais 23 municípios do Estado 232021222331 . Mais recentemente, a presença de B. glabrata foi constatada em municípios do norte do Estado, situados nas bacias hidrográficas dos rios Coruripe, Traipu, Mundaú e Paraíba, onde sucede alta endemicidade da esquistossomose ${ }^{10}$, e ao sul, em Penedo ${ }^{11}$.

A prevalência da doença no Brasil justifica a necessidade de atualização da distribuição dos moluscos vetores nas localidades de risco ${ }^{35}{ }^{36}$. A distribuição real das espécies, todavia, não está bem esclarecida, dificultada pela grande extensão territorial e pela carência de recursos humanos ${ }^{26}$.

Segundo dados da Fundação Nacional de Saúde ( FUNASA), através do Programa de Controle da Esquistossomose em Alagoas, há um recrudescimento da endemia ${ }^{1213}$. De acordo com esses dados, $60 \%$ do território alagoano seria área endêmica e mais de dois milhões de indivíduos estariam expostos à infecção. A presente pesquisa, que abrange 40 dos 102 municípios alagoanos, tem por objetivo a identificação de B. glabrata em duas mesorregiões do Estado de Alagoas, 0 exame dos moluscos capturados para detecção de cercárias do S. mansoni e 0 levantamento da prevalência da esquistossomose nessas áreas.

Mesorregião do Leste Alagoano. a) Vegetação Litorânea/ Zona da Mata: Maragogi, S. Miguel dos Milagres, Passo de Camaragibe, Marechal Deodoro, S. Miguel dos Campos, Piaçabuçu. b) Vegetação Zona da Mata: Atalaia, Igreja Nova, Jacuípe, Jundiá, Colônia Leopoldina, Novo Lino, Ibateguara, S. José da Laje, Santana do Mundaú, União dos Palmares, Joaquim Gomes, Matriz de Camaragibe, S. Luís do Quitunde, Flexeiras, Murici, Branquinha, Satuba, Santa Luzia do Norte, Messias, Pilar, Porto Real do Colégio, Penedo.
Mesorregião do Agreste Alagoano. a) Vegetação Cerrado: Chã Preta, Viçosa, Paulo Jacinto, Junqueiro, São Sebastião, Teotônio Vilela, Campo Alegre, Igaci, Coité do Nóia, Arapiraca, Lagoa da Canoa. b) Vegetação Agreste: Jaramataia.

\section{MATERIAL E MÉTOD0S}

Área de estudo. Os 40 municípios pesquisados pertencem à mesorregião do leste Alagoano (28) e à mesorregião do Agreste Alagoano (12). Predomina o clima tropical quente e úmido, com seca no verão e chuva no outono e inverno $0^{1524}$. Os municípios da Região Leste, em maior número, possuem a vegetação da Zona da Mata Atlântica. A floresta sofre destruição contínua desde 0 início da colonização, substituída por lavouras e pastagens. Além dos rios maiores, há variedades de coleções hídricas como riachos, açudes, poços, valas, lagoas, brejos, córregos, que constituem criadouros perenes da B. glabrata. Esta espécie encontra-se predominantemente em áreas mais úmidas ${ }^{32}$.

Inquérito malacológico. Os planorbídeos foram coletados em águas rasas de coleções hídricas localizadas nas propriedades rurais ou nas valas e canais de escoamento nas zonas urbanas. As coletas foram realizadas por técnicos da FUNASA, no período de fevereiro de 1996 a dezembro de 1998.

Exame dos moluscos. Após o reconhecimento dos criadouros, os moluscos foram levados ao laboratório, contados e examinados. Foram separados três exemplares, em média, de cada amostra para identificação morfológica e 0 restante para identificação de cercárias do S. mansoni por compressão entre placas de vidro. A identificação morfológica foi realizada de acordo com o protocolo estabelecido por Paraense (1975).

Classificação dos tipos de criadouros. Os criadouros foram classificados pelos técnicos da Fundação Nacional de Saúde em riachos, açudes, poços, valas, lagoas, córregos e brejos. Nos criadouros com B. glabrata infectados foi aplicado moluscicida, de acordo com norma estabelecida pela FUNASA.

Inquérito coprológico. Amostras de fezes dos habitantes dos municípios foram coletadas e examinadas pelo método de Kato-Katz ${ }^{17}$. Os exames foram realizados pela FUNASA durante os anos de 1997 a 2000.

\section{RESULTAD0S}

Moluscos. Identificação: os espécimes apresentaram conchas de coloração variada, os maiores mais escuros, marrom-esverdeados e os menores geralmente com a cor marrom-claro. Diâmetros menores ( $7 \mathrm{~mm})$ prevaleceram. A ocorrência de tamanhos diminutos (de até $4,1 \mathrm{~mm}$ ), de conchas vazias ou com material deteriorado impossibilitou, em alguns casos, o procedimento de identificação.

Foi constatada a presença de B. glabrata em 30 (75\%) dos 40 municípios estudados. No leste Alagoano, 20 (71,4\%) municípios apresentaram a espécie: Marechal Deodoro, Piaçabuçu, São Miguel dos Campos, Ibateguara, Joaquim Gomes, Jundiá, Matriz de Camaragibe, Messias, Novo Lino, 
São José da Laje, São Luís do Quitunde, Santana do Mundaú, União dos Palmares, Penedo, Branquinha, Flexeiras, Murici, Pilar, Igreja Nova e Porto Real do Colégio. No Agreste, 10 (83,3\%) dos 12 municípios apresentaram-na: Arapiraca, Coité do Nóia, Igaci, Jaramataia, Lagoa da Canoa, Chã Preta, Junqueiro, Paulo Jacinto, São Sebastião e Campo Alegre.

Infecção. A presença de cercárias do S. mansoni foi constatada em espécimes de seis municípios. Penedo apresentou a maior (6,6\%) taxa de infecção, seguido por Ibateguara ( 5,6\%). Taxas menores foram observadas em Chã Preta (2,7\%), Murici (2,5\%), Porto Real do Colégio (0,1\%) e Igreja Nova (0,1\%) (Tabela 1).

Tabela 1- Taxas de infecção por Schistosoma mansoni em Biomphalaria glabrata e na população local, nos municípios que apresentaram moluscos infectados.

\begin{tabular}{|c|c|c|c|c|}
\hline \multirow[t]{3}{*}{ Municípios } & \multirow{3}{*}{$\begin{array}{c}\text { Moluscos infectados } \\
1996 / 1998 \\
(\%)\end{array}$} & \multicolumn{3}{|c|}{ População afetada } \\
\hline & & 1997 & 1998/1999 & 2000 \\
\hline & & $(\%)$ & $(\%)$ & $(\%)$ \\
\hline Penedo & 6,6 & - & 21,9 & 12,4 \\
\hline Ibateguara & 5,6 & 24,9 & 12,8 & - \\
\hline Chã Preta & 2,7 & 35,4 & - & - \\
\hline Murici & 2,5 & 10,9 & 13,8 & 10,1 \\
\hline Porto Real do Colégio & 0,1 & 27,4 & 8,0 & 5,0 \\
\hline Igreja Nova & 0,1 & 18,8 & 14,0 & 7,8 \\
\hline
\end{tabular}

Criadouros. No leste Alagoano, onde foram pesquisadas 602 localidades, com um total de 1.712 criadouros, prevaleceram os poços $(40,8 \%)$ e em seguida, os riachos (33,3\%). No Agreste Alagoano, em 80 localidades com 220 criadouros, predominaram os açudes (40\%), seguidos dos riachos e poços (24\%).

Exames parasitológicos de fezes. A população local dos municípios endêmicos mostrou alta percentagem de casos positivos. No período de 1997, os exames de 156.470 habitantes de 23 municípios mostraram uma taxa de infecção de $21,2 \%$. Nos anos 1998/1999, foi encontrada taxa de 17,6\% após exame de 288.402 indivíduos de 37 municípios, e no ano 2000, após 98.215 exames de habitantes de 21 municípios, achou-se 12,6\% de infecção. Verificou-se importante redução dessas taxas, resultado que deve levar em conta 0 fato dos exames dos exames não terem sido realizados nas mesmas pessoas.

\section{DISCUSSÃ0}

A presença de B. glabrata em 75\% dos municípios estudados confirma esta espécie como principal vetor da esquistossomose no Estado de Alagoas. Embora não se tenha observado B. glabrata em dez municípios, estudos anteriores relatam sua presença em três deles: Viçosa ${ }^{10}{ }^{21}$, Atalaia ${ }^{1011}{ }^{23}$ e Maragogi ${ }^{2}$.

Na mesorregião do leste Alagoano, onde B. glabrata foi encontrada em 20 dos 28 municípios estudados, verifica-se abundância de poços e depois, de riachos. Poços e riachos não sujeitos à dessecação favorecem o desenvolvimento da espécie ${ }^{32}$.
Na mesorregião do Agreste Alagoano, a presença da espécie foi constatada em dez dos 12 municípios estudados, incluindo Jaramataia, já próxima da região semi-árida. Percebe-se, portanto, que ela pode colonizar habitats sujeitos a secas estacionais, mantendo-se em estivação. Este fato acontece comumente no Nordeste do Brasil, na zona de transição entre a costa úmida e 0 interior semi-árid ${ }^{26}$. Aqui, como se pode prever, os açudes são as coleções de água mais freqüentes.

Em ambas as mesorregiões a predominância de criadouros artificiais, poços e açudes, vêm demonstrar a importância da presença humana na disseminação da $\mathrm{EM}^{9} 103040$.

Foram coletados espécimes com infecção natural pelo S. mansoni em seis municípios. Penedo, no leste Alagoano, apresentou índices mais ( 6,6\%) altos, o que pode ser atribuído à cultura do arroz. Os arrozais, com seus alagados, favorecem a proliferação dos moluscos ${ }^{18}$. Em estudo anterior, foi encontrada neles taxa de infecção mais alta, de 7,3\%, nesse município ${ }^{11}$.

Taxas relevantes (85\%) de infecção natural em B. glabrata foram observadas excepcionalmente em municípios do Estado de Minas Gerais $^{37}$. Porém, consideram-se comuns taxas de positividade baixas, em criadouros naturais, entre 0,5 e 3\% e excepcionalmente são observadas médias de 5 a 25\% em áreas hiperendêmicas ${ }^{40}$. Na zona sul de Belo Horizonte, totalmente urbana, foram efetuadas 28 capturas no período de dez anos (1983 a 1992), quando foram coletados 3.361 exemplares de B. glabrata, dos quais $23(0,7 \%)$ estavam infectados pelo S. mansoni ${ }^{16}$.

Municípios nos quais a FUNASA efetuou exames parasitológicos na população mostraram número importante de indivíduos infectados pelo S. mansoni. № entanto, com a continuação dos inquéritos, verificou-se diminuição desse número, provavelmente em decorrência do tratamento administrado aos casos positivos e do emprego de moluscidas, previstos no programa. Também pode-se considerar que os exames não foram efetuados nos mesmos indivíduos. Na década anterior, a FUNASA constatou que dentre 18 estados, do Nordeste e Sudeste, Alagoas apresentou o mais alto índice de exames positivos ${ }^{40}$.

Fica ressaltada, neste estudo, a importância epidemiológica de B. glabrata como principal transmissora da EM nas zonas endêmicas do Estado de Alagoas. 0 estudo da distribuição geográfica dos moluscos vetores da doença no Brasil deve persistir, mormente nas regiões onde sua importância epidemiológica ainda não está estabelecida. Aliás, vem sendo observado nas últimas décadas, um progresso nos estudos visando melhor conhecimento da fauna planorbídica 5678121314282931343639414243 . 0 conhecimento das espécies, de sua densidade nos criadouros e das taxas de infecção natural serão recursos importantes para uma melhor adequação das medidas preventivas.

\section{AGRADECIMENTOS}

Ao Dr. Wladimir L. Paraense, Chefe do Laboratório de Malacologia, Intituto Oswaldo Cruz/Fundação Oswaldo Cruz, à Dra ${ }^{a}$ iriane M. Freitas e ao Dr. Haroldo da S. Ferreira, da Universidade Federal de Alagoas, pelas sugestões e comentários. Aos técnicos da Fundação Nacional de Saúde, João Florentino dos Santos e Antonio Soares Sobrinho, pela colaboração necessária nesta pesquisa. 


\section{REFERÊNCIAS BIBLIOGRÁFICAS}

1. Barbosa CS, Pieri OS, Silva CB, Barbosa FS. Ecoepidemiologia da esquistossomose urbana na ilha de Itamaracá, Estado de Pernambuco. Revista de Saúde Pública, São Paulo 34: 4, 2000.

2. Barbosa FS, Figueiredo T. Geographical distribution of the snail hosts of schistosomiasis mansoni in Northeastern Brazil. Revista do Instituto de Medicina Tropical de São Paulo 11: 285-289, 1969.

3. Barbosa FS, Pessoa D, Oliveira P, Barbosa JM, Rodrigues BA. Levantamentos seccionais sobre a esquistossomose no Nordeste do Brasil. I. Estado de Alagoas. Revista da Sociedade Brasileira de Medicina Tropical 4:129-152, 1970.

4. Carvalho OS, Massara CL, Guerra HL, Campos YR, Caldeira RL, Chaves A, Katz N. Re-avalution of schistosomiasis mansoni in Minas Gerais- Brazil III. Noroeste de Minas mesoregion. Revista do Instituto de Medicina Tropical de São Paulo 40: 277-279, 1998a.

5. Carvalho OS, Massara CL, Rocha RS, Katz N. Esquistossomose mansoni no sudoeste do Estado de Minas Gerais (Brasil). Revista Saúde Pública 23: 341-344, 1989.

6. Carvalho OS, Massara CL, Silveira Neto HV, Alvarenga AG, Vidigal THDA, Guerra HL, Santos MA, Chaves A, Katz N. Schistosomiasis mansoni in the Region of the Triângulo Mineiro, State of Minas Gerais, Brazil. Memórias do Instituto Oswaldo Cruz 89: 509-512, 1994

7. Carvalho OS, Massara CL, Silveira Neto HV, Guerra HL, Caldeira RL, Mendonça LF, Vidigal THDA, Chaves A, Katz N. Re-evalution of schistosomiasis mansoni in Minas Gerais, Brasil II. Alto Paranaíba Mesoregion. Memórias do Instituto Oswaldo Cruz 92: 141-142, 1997.

8. Carvalho OS, Nunes IM, Caldeira RL. First report of Biomphalaria glabrata in the State of Rio Grande do Sul, Brazil. Memórias do Instituto Oswaldo Cruz 93: 39-40, 1998b.

9. Cha YN, Edwards R. Effect of Schistosoma mansoni infection of the hepatic drug-metabilizing of mice. The Journal of Pharmacology and Experimental Therapeutics 199: 432-440, 1976

10. Couto JLA. Biomphalaria (Mollusca: Pulmonata: Basommatophora) nas Meso-Regiões do Agreste e do Leste Alagoano, Alagoas, Brasil. Taxonomia, considerações ecológicas e epidemiológicas. Monografia. Universidade Federal de Alagoas, Maceió, Alagoas, 1995.

11. Couto JLA. Estudo e identificação de espécies de Biomphalaria transmissores da esquistosomose em três municípios do Estado de Alagoas. In: Resumos do XV Congresso Brasileiro de Parasitologia, Salvador, p. 42, 1997.

12. Fundação Nacional de Saúde. Retrospectiva e Comentários das Operações de Campo do Programa de Controle da Esquistossomose no Estado de Alagoas, no período de 1977 a 1994. Programa de Controle da Esquistossomose. Ministério da Saúde, Alagoas, 1995a.

13. Fundação Nacional de Saúde. Relatório de Atividades. Programa de Controle da Esquistossomose, Ministério da Saúde, Brasília, 1995b.

14. Fundação Nacional da Saúde. Lista dos municípios do Estado de Minas Gerais que apresentam planorbídeos de importância na transmissão da esquistossomose. Gerência técnica de esquistossomose de Brasília, Ministério da Saúde Brasília, 1998.

15. Governo do Estado de Alagoas. Enciclopédia dos Municípios Alagoanos. Maceió, Alagoas, 1977.

16. Guimarães CT, Souza MA, Soares DM, Souza, CP. Levantamento malacológico em parques urbanos de Belo Horizonte, Minas Gerais, Brasil. Cadernos de Saúde Pública 13, 1997.

17. Katz, N, Chaves A, Pellegrino J. A simple device for quantitative stool thick smear technique in schistosomiasis mansoni. Revista do Instituto de Medicina Tropical de São Paulo 14: 397-400, 1972

18. Kloos H, Souza C, Gazzinelli A, Soares Filho BS, Temba PC, Bethony J, Page $\mathrm{K}$, Grzywarz C, Lewis F, Minchella D, Loverde P, Oliveira RC. The distribution of Biomphalaria sp in different habitats in relation to physical, biological water contact and cognitive factors in a rural área in Minas Gerais, Brazil. Memórias do Instituto Oswaldo Cruz, Rio de Janeiro 96: 57-66, 2001.
19. Lucena DT. Mais um foco de schistosomíase mansoni em Alagoas, comprovado pela descoberta de Planorbis infectados. Anais da Sociedade de Biologia de Pernambuco 7: 37-42, 1947.

20. Lucena DT. Segunda lista de moluscos do Nordeste. Boletim da Secretaria de Agricultura, Indústria e Comércio, Pernambuco 16: 126-135, 1949.

21. Lucena DT. Terceira lista de moluscos do Nordeste. Boletim da Secretaria de Agricultura, Indústria e Comércio, Pernambuco 17: 32-51, 1950.

22. Lucena DT. Resenha sistemática dos planorbídeos brasileiros. Gráfica Editora Recife S.A., Recife, 1956

23. Lucena DT. Planorbídeos transmissores da esquistossomose no Nordeste do Brasil. Revista Brasileira de Malariologia e Doenças Tropicais 15: 13-26, 1963.

24. Ministério da Economia, Fazenda e Planejamento. Divisão Regional do Brasil em Mesorregiões e Microrregiões Geográficas. Rio de Janeiro, 1977.

25. Paraense WL. The nomenclature of Brazilian planorbids.III. Australorbis stramineus ( Dunker, 1848). Revista Brasileira de Biologia 23: 1-7, 1963.

26. Paraense WL. Fauna planorbídica do Brasil. In: Blucher E (ed) Introdução à Geografia Médica do Brasil, São Paulo, p. 213-239, 1972.

27. Paraense WL. Estado atual da sistemática dos planorbídeos brasileiros. Arquivos do Museu Nacional 55: 105-111, 1975.

28. Paraense WL. A survey of planorbid mollusks in the Amazonian region of Brazil. Memórias do Instituto Oswaldo Cruz 78: 343-361, 1983.

29. Paraense WL. Distribuição dos caramujos no Brasil. In: Suplemento dos Anais 1983/84, Academia Mineira de Medicina, Belo Horizonte, p. 117-128, 1986.

30. Paraense WL. The schistosome vectors in the Americas. Memórias do Instituto Oswaldo Cruz 96: 7-16, 2001.

31. Paraense WL, Alencar AJT, Correa LR. Distribuição dos planorbídeos e prevalência da xistosomose mansoni no Estado do Espírito Santo. Memórias do Instituto Oswaldo Cruz 78: 374-384, 1983.

32. Pereira G. Esquistossomose mansônica em Pernambuco: consideraç̃oes epidemiológicas. Arquivo Brasileiro de Medicina 60: 357-360, 1986.

33. Pessoa SB, Amorim JP. Contribuição para a história natural da esquistossomose mansônica no nordeste brasileiro e sugestões para sua profilaxia. Revista Brasileira de Malariologia e Doenças Tropicais 9: 5-18, 1957.

34. Souza CP. Estudo dos moluscos do gênero Biomphalaria de Minas Gerais, com relação à adaptação parasito hospedeiro e importância na epidemiologia da esquistossomose. Revista do Instituto de Medicina Tropical de São Paulo 28: 287-292, 1986.

35. Souza CP, Caldeira RL, Drummond SC, Melo AL, Guimarães CT, Soares DM, Carvalho OS. Geographical distribution of Biomphalaria snails in State of Minas Gerais, Brazil. Memórias do Instituto Oswaldo Cruz 96: 293-302, 2001.

36. Souza CP, Drummond SC, Silva CJE, Queiroz LA, Guimarães CT, Rocha RC. Investigação sobre a transmissão da esquistossomose no complexo turístico da Serra do Cipó, M.G. Informe Epidemiológico SUS 4: 43-51, 1998 a.

37. Souza CP, Jannotti-Passos LK, Coura-Filho P. Risk of transmission of schistosomiasis in the Parque Estadual do Rio Doce, M.G. (Brazil) and its surrounds. II: Malacological Survey in Dionísio, M.G. In: Resumos do VI International Symposium on Schistosomiasis, Belo Horizonte, p. 136, 1997.

38. Souza CP, Jannotti-Passos LK, Freitas JR. Degree of host-parasite compatibility between Schistosoma mansoni and their intermediate molluscan hosts in Brazil. Memórias do Instituto Oswaldo Cruz 90: 5-10, 1995.

39. Souza CP, Lima LC, Jannotti-Passos LK, Ferreira SS, Guimarães CT, Vieira IBF, Mariani-Junior R R. Moluscos límnicos da microrregião de Belo Horizonte, M.G., com ênfase nos vetores de parasitoses. Revista da Sociedade Brasileira de Medicina Tropical 31: 449-456, $1998 \mathrm{~b}$.

40. Rey L. Bases da Parasitologia Médica. Guanabara-Koogan, Rio de Janeiro, 1992.

41. Teles HMS. Distribuição de Biomphalaria straminea (Dunker, 1848) (Pulmonata, Planorbidae) no Estado de São Paulo (Brasil). Ciência e Cultura 40: 508-512, 1987

42. Teles HMS. Distribuição de Biomphalaria tenagophila e B. occidentalis no Estado de São Paulo (Brasil). Revista de Saúde Pública 23: 244-253, 1989.

43. Teles HMS. Distribuição de Biomphalaria straminea ao sul da região neotropical, Brasil. Revista de Saúde Pública 30: 341-349, 1996. 